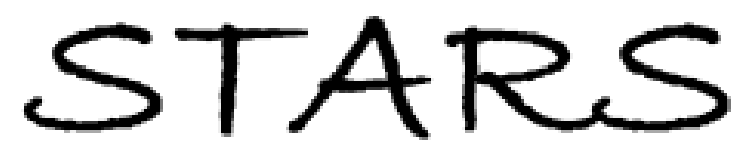

University of Central Florida

STARS

Faculty Bibliography 2010s

Faculty Bibliography

$1-1-2010$

\title{
Cassini Uvis Stellar Occultation Observations of Saturn's Rings
}

\author{
J. E. Colwell \\ University of Central Florida \\ L. W. Esposito \\ R. G. Jerousek \\ University of Central Florida \\ M. Sremčević \\ D. Pettis \\ University of Central Florida
}

See next page for additional authors

Find similar works at: https://stars.library.ucf.edu/facultybib2010

University of Central Florida Libraries http://library.ucf.edu

This Article is brought to you for free and open access by the Faculty Bibliography at STARS. It has been accepted for inclusion in Faculty Bibliography 2010 s by an authorized administrator of STARS. For more information, please contactSTARS@ucf.edu.

\section{Recommended Citation}

Colwell, J. E.; Esposito, L. W.; Jerousek, R. G.; Sremčević, M.; Pettis, D.; and Bradley, E. T., "Cassini Uvis Stellar Occultation Observations of Saturn's Rings" (2010). Faculty Bibliography 2010s. 61.

https://stars.library.ucf.edu/facultybib2010/61

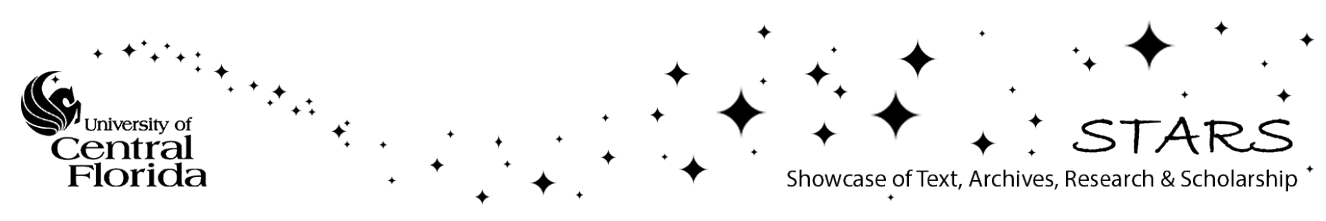




\section{Authors}

J. E. Colwell, L. W. Esposito, R. G. Jerousek, M. Sremčević, D. Pettis, and E. T. Bradley 


\title{
CASSINI UVIS STELLAR OCCULTATION OBSERVATIONS OF SATURN'S RINGS
}

\author{
J. E. Colwell ${ }^{1,3}$, L. W. Esposito ${ }^{2}$, R. G. Jerousek ${ }^{1}$, M. Sremčević ${ }^{2}$, D. Pettis ${ }^{1}$, And E. T. Bradley ${ }^{1}$ \\ ${ }^{1}$ Department of Physics, University of Central Florida, Orlando, FL 32816-2385, USA; jcolwell@ mail.ucf.edu \\ 2 LASP, University of Colorado, 392 UCB, Boulder, CO 80309-0392, USA \\ Received 2010 April 28; accepted 2010 September 7; published 2010 October 20
}

\begin{abstract}
The Cassini spacecraft's Ultraviolet Imaging Spectrograph (UVIS) includes a high-speed photometer (HSP) that has observed more than 100 stellar occultations by Saturn's rings. Here, we document a standardized technique applied to the UVIS-HSP ring occultation datasets delivered to the Planetary Data System as higher level data products. These observations provide measurements of ring structure that approaches the scale of the largest common ring particles $(\sim 5 \mathrm{~m})$. The combination of multiple occultations at different viewing geometries enables reconstruction of the three-dimensional structure of the rings. This inversion of the occultation data depends on accurate calibration of the data so that occultations of different stars taken at different times and under different viewing conditions can be combined to retrieve ring structure. We provide examples of the structure of the rings as seen from several occultations at different incidence angles to the rings, illustrating changes in the apparent structure with viewing geometry.
\end{abstract}

Key words: instrumentation: photometers - methods: observational - planets and satellites: rings - space vehicles: instruments

Online-only material: color figure

\section{INTRODUCTION}

We present a detailed description of a systematic processing of Cassini Ultraviolet Imaging Spectrograph (UVIS) stellar occultation data. Stellar and radio occultations by Saturn's rings provide the highest resolution profiles of ring structure. The Cassini spacecraft has three instruments that observed high-resolution occultations of the rings: UVIS and the Visual and Infrared Mapping Spectrometer (VIMS) which observe occultations of ultraviolet and infrared stars, respectively, and the Radio Science Subsystem (RSS) which transmits a coherent radio signal through the rings to the Earth. These datasets have revealed evidence for viscous overstability oscillations (Thomson et al. 2007), shown the characteristics of temporary agglomerations of ring particles (Colwell et al. 2006, 2007; Hedman et al. 2007a), revealed small clumps in the $\mathrm{F}$ ring (Esposito et al. 2008), and resolved sharp ring edges (Colwell et al. 2008) and short-wavelength waves throughout the rings (Colwell et al. 2009).

Combining multiple occultation profiles from different observing geometries enables reconstruction of the threedimensional structure of the rings (e.g., Colwell et al. 2006, 2007). This inversion of multiple occultations to obtain information about ring structure is analogous to computed tomography scans in medical imagery with one critical difference: the occultations are widely spaced in time. These data must be appropriately calibrated so that there are no systematic offsets between observations that could be confused with ring structure. In the case of the UVIS High-Speed Photometer (HSP) this involves removal of the background (non-stellar) signal from the data and compensation for a drift in the instrument's sensitivity during the course of an occultation (Colwell et al. 2007).

Cassini observations have revealed the Saturn ring system to be particularly dynamic, with structural changes observed on timescales of years. The structure of the innermost D ring changed significantly between the Voyager flybys and the arrival of Cassini (Hedman et al. 2007b). The F ring has undergone fundamental structural changes even during the

\footnotetext{
3 Author to whom any correspondence should be addressed.
}

time Cassini has been in orbit (Albers et al. 2010; Charnoz et al. 2005; Murray et al. 2005), and ephemeral clumps have been observed in the $\mathrm{F}$ ring in images (Murray et al. 2008; Beurle et al. 2010) and occultations (Esposito et al. 2008). There are also many clues to the evolution of the remaining main rings ( $\mathrm{A}, \mathrm{B}, \mathrm{C}$, and the Cassini Division) on multiple timescales. The shifting orbits of nearby moons, notably the co-orbital satellites Janus and Epimetheus and the F ring moons Prometheus and Pandora, result in changes to the locations of resonances and the ring structures, primarily density waves, associated with them (e.g., French et al. 2003; Tiscareno et al. 2007). On arrival at Saturn, Cassini discovered evidence of small moonlets, dubbed propeller objects for the characteristic perturbations they produce in the surrounding ring material, in the outer A ring (Tiscareno et al. 2006). The distribution of these moonlets is non-uniform, suggesting a possible origin in the fragmentation of a small number of larger objects (Sremčević et al. 2007; Tiscareno et al. 2008). Serendipitous passes of stellar occultation paths across clumps, moonlets, or their associated disturbances in the nearby ring material, provide high-resolution one-dimensional measurements of structure on the scale of the largest ring particles.

In Section 2, we describe the UVIS stellar occultation data including a tabular summary of all occultations observed to date. In Section 3, we describe a standard procedure for the calibration of the occultation data used to generate the optical depth profiles delivered to the Planetary Data System (PDS). We present examples of these optical depth profiles and discuss the uncertainties in the calibration.

\section{OBSERVATIONS}

The Cassini UVIS observes stellar occultations by the rings through two channels: the HSP, and the Far Ultraviolet (FUV) spectrograph. The HSP is the primary channel for measuring ring structure because of its fast sampling time of 1-8 ms. The FUV channel is used for simultaneous measurements of the UV ring spectral reflectance as well as for imaging the star and rings to facilitate determination of background levels in the HSP data. The HSP and FUV have the same spectral bandpass 
of approximately 110-190 nm (Esposito et al. 2004), while the FUV has 1024 spectral resolution elements and 64 spatial resolution elements along the slit. A single FUV integration produces much more data than the HSP which is typically operated in a 9 bit compression mode. The FUV is less sensitive than the HSP and cannot be read out as quickly, so it is not used for measurements of ring structure. However, the imaging capabilities of the FUV do allow simultaneous measurements of the ring brightness in the same part of the spectrum that the HSP measures. Due to data rate limitations, the integration period for these simultaneous FUV observations is typically at least $60 \mathrm{~s}$ compared to 1 or $2 \mathrm{~ms}$ for the HSP. Although the sensitivities of both the FUV and HSP are changing in different ways as the mission progresses, the total FUV sensitivity to $\alpha$ Vir was $\sim 20,000$ counts $\mathrm{s}^{-1}$ on the Rev 030 occultation compared to over 500,000 counts $\mathrm{s}^{-1}$ observed in the HSP (Table 1). For some occultations, the FUV data can be used in constructing a model of the background component of the HSP signal (Section 3.3; see also Colwell et al. 2007). Because this process depends on additional models of the instrument response and the azimuthal behavior of ring brightness, we do not use the FUV data in determining the background provided to the PDS; instead we provide the FUV data so that interested users can create a new background model making use of this additional information.

The photon count rate, $I$, measured by the HSP includes light from the star and a background photon count rate, $b$, and depends on the transparency, $T$, of the intervening ring material. The background has contributions from interplanetary Ly $\alpha$ emission and sunlight reflected off the rings. The latter component of the background is highly sensitive to the occultation viewing geometry and dominates $b$ for observations of the sunlit face of the rings. The interplanetary Ly $\alpha$ signal is the dominant source of background when the unlit face of the rings is observed. It, too, varies over the course of the occultation due to the different transparency of the rings at different times in the occultation, allowing more or less Ly $\alpha$ to shine through the rings. A direct measurement of $b$ is only possible when there is no signal from the star. This is typically only the case when the star is behind the opaque central $B$ ring, though for fainter stars or occultations at a low elevation angle above the ring plane, $B$, the star is not detected at other locations. If the measured photon count rate due to the unocculted star alone is $I_{0}$, then the measured photon count rate is given by

$$
I=I_{0} T+b .
$$

The count rate due to the unocculted star, $I_{0}$, is measured in regions where $T=1$ and $I=I_{0}+b$. With the background determined from measurements where $T=0$ and $I=b, I_{0}$ can be determined. Tables 2 and 3 list the locations of regions we use to determine $b$ and $I_{0}$.

Ignoring stellar variation, $I_{0}$ is ideally constant and a single measurement either in a ring gap or outside the rings would be sufficient to determine it for the entire occultation, leaving all the uncertainties in $b$. While stellar variability is not a significant source of variation or uncertainty in $I_{0}$ for any of the UVIS occultations, the HSP does not have a constant response to bright signals. This results in variations in the measured value of $I_{0}$ over the course of an occultation so that it can only be precisely determined (ignoring for the moment uncertainties in $b$ ) in the sparsely spaced gaps in the rings. The nature of the HSP response to bright signals is shown in Figure 1. When exposed to a bright star, the photon count rate measured by the HSP increases rapidly for several minutes and then increases more

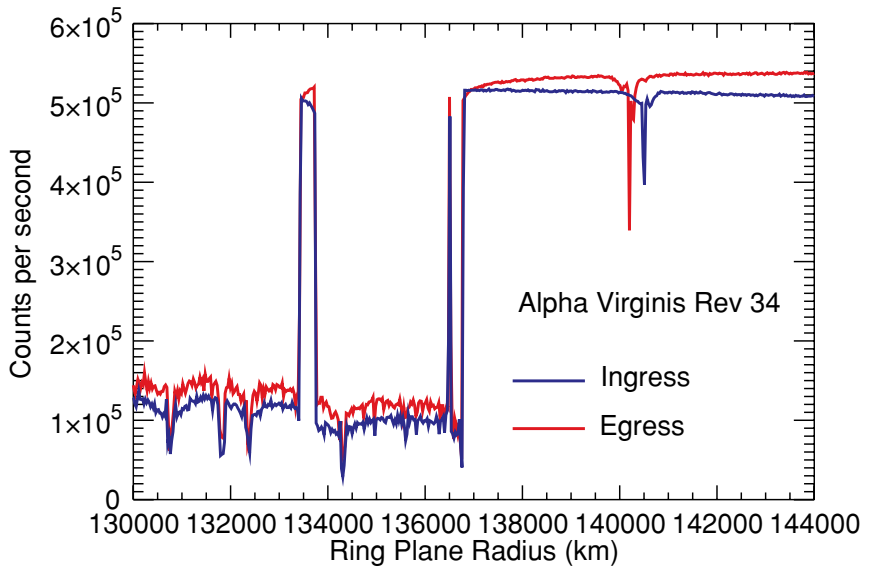

Figure 1. Outer A ring region and the $\mathrm{F}$ ring in the ingress (blue) and egress (red) portions of a chord occultation of the star $\alpha$ Virginis. The Encke Gap at $133,500 \mathrm{~km}$ illustrates the change in instrument response as a function of its history of exposure. The signal is low when it first crosses the Encke gap edge and gradually increases while it is behind the gap. The instrumental response is also responsible for the offset between the ingress and egress light curves in the region beyond the outer edge of the A ring at $136,750 \mathrm{~km}$. The difference in signal in the A ring is not due to instrumental effects: just inside the gap values of $I_{0}$ for both ingress and egress are equal, so the instrument sensitivity is equal at this location for both ingress and egress. Just interior to the inner edge (where $I_{0}$ is observed to be the same) values of $I$ are different, and this must therefore not be due to instrumental effects.

gradually. When the star passes behind a gap in the rings, the photon count rate is initially lower than when the star was last previously observed unocculted and then begins to gradually increase again (see also Colwell et al. 2007). The value of $I$ is different when the star is first occulted by a ring and when it emerges from the ring. Without a validated model of the instrument's nonlinear behavior, it is therefore not possible to know what the value of $I_{0}$ would be at each point when the star is behind the ring more precisely than the difference in the observed values of $I_{0}$ on either side of the ring. The estimated value of the star photon count rate, $I_{0}$, is used to calculate the slant path optical depth,

$$
\tau=\ln (1 / T)=\ln \left(\frac{I_{0}}{\max \left[I-b, I_{\min }\right]}\right),
$$

where the denominator is the maximum value of $I-b$ and a selected minimum detectable photon count rate, $I_{\min }$.

Before Equation (2) can be used, the uncertainties in $I$ and $b$ must be estimated, in part to place a lower detectable limit, $I_{\min }$, on the quantity $I-b$. In practice, $I-b$ can be negative due to statistical variations in the data and systematic uncertainties in $b$. Placing a non-zero minimum value on $I-b$ is equivalent to specifying a maximum normal optical depth that can be distinguished from infinite optical depth, $\tau_{\max }$. In practice, we determine $\tau_{\max }$ based on counting statistics as described in Section 3.3 and calculate the normal (perpendicular to the ring plane) optical depth using $\tau_{\max }$ instead of $I_{\min }$ :

$$
\tau_{n}=\min \left[\mu \ln \left(\frac{I_{0}}{I-b}\right), \tau_{\max }\right]
$$

where $\mu=\sin (B)$ with $B$ being the angle between the ring plane and the line of sight from the spacecraft to the star. The normal optical depth at a particular location in the rings varies with the azimuthal viewing geometry due to self-gravity wakes, so that even occultations of the same star yield different optical depth 
Table 1

Occultation Summary

\begin{tabular}{|c|c|c|c|c|c|c|}
\hline Occultation Star (rev) Side & Date (Year-Day) & $|B|(\operatorname{deg})$ & $\phi \operatorname{deg})$ & $R(\mathrm{~km})$ & Duration (s) & $I_{0}(\mathrm{~Hz})$ \\
\hline$\xi 2 \operatorname{Cet}(\mathrm{A}) \mathrm{E}$ & 2004-280 & 14.9 & $72.9-89.4$ & $57,757-80,239$ & 27197 & 1,300 \\
\hline$\xi 2 \operatorname{Cet}(\mathrm{A}) \mathrm{E}$ & 2004-281 & 14.9 & $98.8-103.8$ & $108,956-135,650$ & 26199 & 1,400 \\
\hline 126 Tau (8) E & 2005-139 & 21.1 & $130.2-88.8$ & $70,380-141,390$ & 31888 & 3,600 \\
\hline$\alpha \operatorname{Vir}(8) \mathrm{I}$ & 2005-141 & 17.2 & $116.1-150.2$ & $118,979-141,954$ & 2546 & 479,000 \\
\hline$\alpha \operatorname{Vir}(8) \mathrm{E}$ & 2005-141 & 17.2 & $116.1-82.2$ & $118,979-141,704$ & 2535 & 509,000 \\
\hline$\delta$ Aqr (8) E & $2005-141$ & 12.2 & $106.8-131.4$ & $60,687-169,884$ & 9871 & 700 \\
\hline$\alpha$ Leo (9) I & $2005-159$ & 9.5 & $68.0-10.7$ & $114,150-204,718$ & 6948 & 46,500 \\
\hline$\alpha$ Leo (9) E & $2005-159$ & 9.5 & $68.0-98.4$ & $114,150-131,539$ & 2663 & 43,200 \\
\hline 126 Tau (10) I & $2005-175$ & 21.1 & $204.3-216.5$ & $103,210-144,810$ & 15898 & 4,100 \\
\hline$\sigma \operatorname{Sgr}(11) \mathrm{I} \#$ & 2005-195 & 29.1 & $221.9-248.8$ & $85,987-146,929$ & 5721 & 117,000 \\
\hline$\alpha \operatorname{Sco} \mathrm{B}(13) \mathrm{I}$ & $2005-232$ & 32.2 & $155.0-208.4$ & $101,173-155,751$ & 6873 & 3600 \\
\hline$\alpha \mathrm{Sco} B(13) \mathrm{E}$ & 2005-232 & 32.2 & $155.1-105.8$ & $101,173-146,576$ & 6027 & 3600 \\
\hline$\zeta$ Oph (26) E & 2006-206 & 16.2 & $126.7-116.6$ & $120,941-149,225$ & 6611 & 28,000 \\
\hline$\lambda$ Cet (28) I & $2006-256$ & 15.3 & $304.0-258.5$ & $74,330-144,011$ & 8901 & 2,500 \\
\hline$\alpha \operatorname{Sco} B(29) \mathrm{I}$ & 2006-269 & 32.2 & $327.3-274.2$ & $79,864-149,436$ & 17154 & 3,500 \\
\hline$\lambda \operatorname{Sco}(29) \mathrm{E} \#$ & $2006-269$ & 41.7 & $189.1-136.4$ & $88,478-143,801$ & 23518 & 285,000 \\
\hline$\alpha \operatorname{Vir}(30) \mathrm{I}$ & $2006-285$ & 17.2 & $266.2-219.8$ & $64,022-151,545$ & 4772 & 535,000 \\
\hline$\gamma \operatorname{Lup}(30) \mathrm{I} \#$ & 2006-286 & 47.4 & $157.1-185.9$ & $83,062-94,587$ & 7467 & 80,000 \\
\hline$\gamma \operatorname{Lup}(30) \mathrm{E} \#$ & 2006-286 & 47.4 & $157.1-102.7$ & $83,062-141,044$ & 18845 & 80,200 \\
\hline$\varepsilon \operatorname{Mic}(30) \mathrm{E}$ & 2006-292 & 31 & $189.1-174.6$ & $97,363-140,210$ & 16382 & $300^{*}$ \\
\hline$\mu$ PsA (31) I & 2006-306 & 30.4 & $248.1-284.2$ & $116,605-144,033$ & 20599 & 800 \\
\hline$\mu$ PsA (31) E & 2006-306 & 30.4 & $248.1-234.8$ & $116,605-119,797$ & 6691 & 700 \\
\hline$\gamma \operatorname{Peg}(32) \mathrm{I}$ & 2006-311 & 20.3 & $110.7-149.5$ & $103,925-155,444$ & 7712 & 75,000 \\
\hline$\gamma \operatorname{Lup}(32) \mathrm{E} \#$ & 2006-313 & 47.4 & $26.3-38.2$ & $84,310-136,192$ & 7046 & 74,200 \\
\hline$\alpha$ Ara (32) I & 2006-314 & 54.4 & $280.9-276.5$ & $61,333-139,786$ & 15231 & 38,900 \\
\hline$\mu$ PsA (32) I & 2006-318 & 30.4 & $248.1-282.6$ & $118,450-143,457$ & 19683 & 700 \\
\hline$\mu$ PsA (32) E & 2006-318 & 30.4 & $248.1-245.2$ & $118,450-118,607$ & 1486 & 500 \\
\hline$\alpha \operatorname{Ara}(33) \mathrm{I}$ & $2006-325$ & 54.4 & $280.8-276.6$ & $65,886-145,356$ & 15461 & 38,700 \\
\hline$\alpha \operatorname{Vir}(34) \mathrm{I}$ & 2006-337 & 17.2 & $282.1-220.9$ & $74,536-153,654$ & 4061 & 506,000 \\
\hline$\alpha \operatorname{Vir}(34) \mathrm{E}$ & 2006-337 & 17.2 & $282.1-344.6$ & $74,536-160,059$ & 4281 & 516,000 \\
\hline$\eta$ Lup (34) I & 2006-337 & 51 & $325.0-286.1$ & $106,848-135,360$ & 10858 & 47,600 \\
\hline$\eta$ Lup (34) E & 2006-337 & 51 & $325.0-7.9$ & $106,848-143,838$ & 12513 & 46,300 \\
\hline$\kappa$ Cen (35) E \# & 2006-350 & 48.5 & $108.9-76.6$ & $68,853-146,169$ & 16765 & 47,100 \\
\hline$\alpha \operatorname{Ara}(35) \mathrm{I}$ & 2006-351 & 54.4 & $221.2-252.0$ & $64,857-130,424$ & 22626 & 37,900 \\
\hline$\alpha \operatorname{Ara}(35) \mathrm{E}$ & 2006-352 & 54.4 & $120.1-113.2$ & $126,867-173,467$ & 13891 & 37,900 \\
\hline$\gamma$ Peg (36) I & 2006-363 & 20.3 & $101.6-156.6$ & $102,296-178,178$ & 9939 & 73,000 \\
\hline$\alpha \operatorname{Peg}(36) \mathrm{E}$ & 2006-363 & 20.3 & $101.6-55.7$ & $102,296-146,785$ & 7172 & 70,100 \\
\hline$\delta$ Per (36) E & 2006-364 & 54 & $68.3-65.7$ & $66,531-140,886$ & 8312 & 13,900 \\
\hline$\kappa$ Cen (36) I \# & 2007-002 & 48.5 & $250.0-237.8$ & $63,531-156,380$ & 18341 & 44,200 \\
\hline$\varepsilon \operatorname{Lup}(36) \mathrm{E} \#$ & 2007-003 & 51 & $36.7-48.3$ & $63,450-148,837$ & 18431 & 33,300 \\
\hline$\alpha \operatorname{Ara}(36) \mathrm{I}$ & 2007-005 & 54.4 & $3.2-311.6$ & $70,897-113,954$ & 24719 & 37,500 \\
\hline$\alpha \operatorname{Ara}(36) \mathrm{E}$ & 2007-005 & 54.4 & $3.2-55.5$ & $70,897-115,410$ & 25228 & 35,700 \\
\hline$\gamma$ Gru (37) I & 2007-009 & 35.1 & $244.3-265.8$ & $137,190-147,326$ & 11069 & 7,300 \\
\hline$\gamma$ Gru (37) E & 2007-009 & 35.1 & $244.3-219.6$ & $137,190-150,985$ & 12999 & 6,800 \\
\hline$\delta$ Per (37) I & $2007-015$ & 54 & $281.1-258.2$ & $60,054-142,584$ & 11273 & 13,700 \\
\hline$\varepsilon$ Lup (37) I \# & 2007-020 & 51 & $324.8-284.8$ & $99,479-129,588$ & 17628 & 31,700 \\
\hline$\varepsilon$ Lup (37) E \# & 2007-020 & 51 & $324.8-10.9$ & $99,479-142,934$ & 21790 & 31,500 \\
\hline$\gamma \operatorname{Ara}(37) \mathrm{I}$ & 2007-022 & 61 & $245.6-251.2$ & $121,471-147,952$ & 7591 & 25,400 \\
\hline$\gamma \operatorname{Ara}(37) \mathrm{E}$ & $2007-022$ & 61 & $142.5-117.2$ & $80,500-155,599$ & 22962 & 27,300 \\
\hline$\varepsilon$ PsA (38) I & $2007-027$ & 23.7 & $255.1-299.3$ & $82,195-114,638$ & 8817 & 2,600 \\
\hline$\varepsilon$ PsA (38) E & $2007-027$ & 23.7 & $255.1-239.1$ & $82,195-85,527$ & 2610 & 2,600 \\
\hline$\psi$ Cen (38) I & 2007-038 & 44.3 & $260.3-243.8$ & $96,426-150,172$ & 12338 & 1,100 \\
\hline$\gamma \operatorname{Ara}(38) \mathrm{I}$ & $2007-041$ & 61 & 212.6-218.7 & $87,186-93,028$ & 2860 & 24,400 \\
\hline$\varepsilon$ PsA (39) I & $2007-045$ & 23.7 & $255.0-277.4$ & $86,910-94,013$ & 3534 & 2,500 \\
\hline$\varepsilon$ PsA (39) E & $2007-045$ & 23.7 & $255.0-233.6$ & $86,910-93,340$ & 3357 & 2,300 \\
\hline$\delta \operatorname{Per}(39) \mathrm{I}$ & $2007-049$ & 54 & $284.1-257.8$ & $55,505-143,284$ & 13131 & 12,600 \\
\hline$\chi$ Cen (39) I & $2007-056$ & 47.5 & $159.9-183.9$ & $98,599-148,429$ & 16186 & 13,200 \\
\hline$\theta$ Ara (40) I & 2007-061 & 53.9 & $16.3-348.5$ & $130,041-146,853$ & 17211 & 15,300 \\
\hline$\theta$ Ara (40) E & 2007-061 & 53.9 & $16.3-33.6$ & $130,041-136,092$ & 10120 & 15,000 \\
\hline$\gamma$ Gru (40) E & $2007-063$ & 61 & $232.3-181.8$ & $67,340-147,704$ & 14990 & 7,500 \\
\hline$\beta$ PsA (40) I & $2007-063$ & 29.2 & $269.5-288.1$ & $119,978-142,564$ & 4846 & 600 \\
\hline 3 Cen (40) E & 2007-073 & 39.3 & $39.4-37.8$ & $110,987-147,354$ & 7481 & 5,000 \\
\hline$\psi$ Cen (40) I & 2007-073 & 44.3 & $216.5-216.9$ & $136,109-149,973$ & 3736 & 2,500 \\
\hline$\theta$ Ara (41) E & 2007-078 & 53.9 & $63.8-89.4$ & $63,681-152,229$ & 25676 & 12,100 \\
\hline$\beta$ Sgr (41) I & 2007-079 & 46.3 & $38.3-18.2$ & $127,360-135,501$ & 9616 & 2,700 \\
\hline$\beta \operatorname{Sgr}(41) \mathrm{E}$ & 2007-079 & 46.3 & $38.3-64.6$ & $127,360-141,957$ & 13040 & 2,900 \\
\hline
\end{tabular}


Table 1

(Continued)

\begin{tabular}{|c|c|c|c|c|c|c|}
\hline Occultation Star (rev) Side & Date (Year-Day) & $|B|(\mathrm{deg})$ & $\phi \operatorname{deg})$ & $R(\mathrm{~km})$ & Duration (s) & $I_{0}(\mathrm{~Hz})$ \\
\hline$\gamma$ Gru (41) I & $2007-080$ & 35.1 & $243.1-294.4$ & $91,528-145,958$ & 13345 & 8,100 \\
\hline$\gamma$ Gru (41) E & $2007-080$ & 35.1 & $243.1-193.0$ & $91,528-142,229$ & 12781 & 7,800 \\
\hline$\delta$ Per (41) I & $2007-082$ & 54 & $232.8-240.6$ & $49,260-149,746$ & 10051 & 12,300 \\
\hline$\kappa$ Cen (42) I \# & 2007-092 & 48.5 & $141.6-178.0$ & $114,712-142,152$ & 27744 & 40,900 \\
\hline$\kappa$ Cen (42) E \# & 2007-092 & 48.5 & $141.6-115.3$ & $114,712-127,824$ & 18632 & 41,700 \\
\hline$\beta$ Per (42) I & 2007-098 & 47.4 & $227.8-230.8$ & $84,461-149,674$ & 5326 & 19,700 \\
\hline$\zeta$ Per (42) I & $2007-098$ & 38 & $328.0-318.1$ & $132,811-134,700$ & 1834 & 10,100 \\
\hline$\zeta$ Per (42) E & $2007-098$ & 38 & $328.0-344.0$ & $132,811-137,957$ & 3046 & 10,100 \\
\hline$\mu$ Sco (43) E \# & $2007-112$ & 43.4 & $155.8-162.1$ & $117,000-123,000$ & 4171 & 76,900 \\
\hline$\lambda \operatorname{Sco}(44) \mathrm{I} \#$ & $2007-129$ & 41.7 & $211.1-244.4$ & $69,544-141,063$ & 19151 & 250,000 \\
\hline$\zeta$ Ori (47) E \# & $2007-179$ & 2.66 & $106.6-99.3$ & $78,770-133,732$ & 3890 & 180,000 \\
\hline$\alpha \operatorname{Sco} B(55) \mathrm{E}$ & 2008-003 & 32.2 & $49.6-68.0$ & $115,023-142,164$ & 7841 & 2,400 \\
\hline$\iota$ Cen (56) E & 2008-014 & 42.7 & $80.0-77.5$ & $131,856-141,213$ & 2880 & $853^{*}$ \\
\hline$v$ Cen (57) I (a) & $2008-026$ & 48 & $167.1-174.2$ & $115,269-122,933$ & 3740 & 38,300 \\
\hline$v$ Cen (57) I (b) & $2008-026$ & 48 & $142.2-146.3$ & $104,915-105,173$ & 1682 & 38,300 \\
\hline$v$ Cen $(57) \mathrm{E}$ & 2008-026 & 48 & $142.2-133.8$ & $104,915-106,010$ & 3479 & 38,300 \\
\hline SAO205839 (57) I & 2008-026 & 15.3 & $263.7-262.7$ & $129,999-146,087$ & 3331 & 11,200 \\
\hline$\iota$ Cen (57) E & $2008-026$ & 52.5 & $80.8-76.6$ & $128,120-144,648$ & 5072 & $945^{*}$ \\
\hline$\kappa$ Cen (57) I \# & $2008-026$ & 48.5 & $238.4-239.3$ & $139,320-146,914$ & 1491 & 20,500 \\
\hline$\beta \operatorname{Lup}(57) \mathrm{I}$ & $2008-026$ & 49.6 & $226.2-231.9$ & $119,418-148,147$ & 5906 & 71,200 \\
\hline$\delta$ Lup (57) I & $2008-026$ & 47 & $259.3-260.4$ & $114,919-147,965$ & 5805 & 48,300 \\
\hline$\gamma \operatorname{Lup}(57) \mathrm{I} \#$ & $2008-026$ & 47.4 & $261.8-262.3$ & $135,464-148,220$ & 2141 & 54,200 \\
\hline$\gamma \mathrm{Cnc}(58) \mathrm{E}$ & $2008-040$ & 21.3 & $227.9-189.4$ & $77,203-160,536$ & 9156 & 2,800 \\
\hline$\beta$ Hya (60) I \# & $2008-058$ & 38.6 & $173.7-183.2$ & $121,736-162,267$ & 12341 & 1000 \\
\hline$\zeta$ Cen $(60)$ I & $2008-060$ & 53.6 & $221.1-231.2$ & $66,648-146,507$ & 17320 & 107,000 \\
\hline$\delta$ Per $(60) \mathrm{I}$ & $2008-062$ & 54 & 283.9-274.5 & $54,975-146,216$ & 7570 & 11,600 \\
\hline$\zeta$ Cen (62) E & 2008-082 & 53.6 & $77.3-67.0$ & $63,689-145,087$ & 17831 & 107,000 \\
\hline$\alpha \operatorname{Ara}(63) \mathrm{E}$ & 2008-092 & 54.4 & $95.8-112.3$ & $73,261-141,566$ & 8531 & 2,900 \\
\hline$\alpha \operatorname{Sex}(63) \mathrm{I}$ & 2008-095 & 2.7 & $271.2-207.7$ & $90,296-202,539$ & 2958 & 500 \\
\hline$\alpha \operatorname{Sex}(63) \mathrm{E}$ & 2008-095 & 2.7 & $271.2-337.4$ & $90,296-223,667$ & 3339 & 500 \\
\hline$\delta$ Cen (64) I \# & $2008-100$ & 55.6 & $117.2-127.9$ & $131,696-133,946$ & 5443 & 63,000 \\
\hline$\delta$ Cen (64) E \# & $2008-100$ & 55.6 & $117.2-107.9$ & $131,696-133,387$ & 4717 & 66,000 \\
\hline$\beta$ Cen (64) E \# & $2008-101$ & 66.7 & $137.8-89.7$ & $84,952-151,166$ & 20690 & 600,000 \\
\hline$\gamma$ Cas (64) I & 2008-102 & 66.3 & 177.9-201.9 & $71,735-119,601$ & 4411 & 103,000 \\
\hline$\varepsilon$ Cen (65) I & $2008-110$ & 59.6 & $221.7-229.0$ & $69,876-148,192$ & 15730 & 130,000 \\
\hline$\alpha \operatorname{Ara}(65) \mathrm{E}$ & $2008-111$ & 54.4 & $110.4-112.9$ & $125,009-143,844$ & 2325 & 25,100 \\
\hline$\delta$ Cen (66) I \# & $2008-119$ & 55.6 & $117.4-142.4$ & $130,072-143,054$ & 13255 & 50,500 \\
\hline$\delta$ Cen (66) E \# & $2008-119$ & 55.6 & $117.4-110.6$ & $130,072-130,974$ & 3416 & 41,600 \\
\hline$\delta$ Cen (68) I \# & $2008-137$ & 55.6 & 201.9-203.4 & $124,551-150,366$ & 4731 & 46,500 \\
\hline$\theta$ Hya (70) I & $2008-156$ & 1.4 & $89.5-17.9$ & $126,499-401,041$ & 3428 & 3,600 \\
\hline$\theta$ Hya (70) E & $2008-156$ & 1.4 & $89.5-160.3$ & $126,499-384,109$ & 3267 & 2,800 \\
\hline$\theta$ Hya (71) I & $2008-163$ & 1.4 & $89.5-23.2$ & $124,439-310,043$ & 2561 & 4,000 \\
\hline$\theta$ Нya (71) E & $2008-163$ & 1.4 & $89.5-153.9$ & $124,439-287,654$ & 2339 & 3,100 \\
\hline$\beta$ Cen (75) I \# & $2008-188$ & 66.7 & $283.5-264.4$ & $72,427-144,448$ & 9611 & 592,000 \\
\hline$\gamma \mathrm{Cnc}(75) \mathrm{I}$ & 2008-190 & 21.3 & $79.8-24.1$ & $71,827-130,700$ & 10241 & 4,400 \\
\hline$\beta$ Cen (77) I \# & 2008-202 & 66.7 & $282.9-264.4$ & $73,334-144,893$ & 9481 & 583,000 \\
\hline$\beta$ Cen (77) E \# & 2008-203 & 66.7 & $34.6-54.4$ & $73,267-143,444$ & 10191 & 604,000 \\
\hline$\beta$ Cen (78) E \# & $2008-210$ & 66.7 & $23.7-54.8$ & $58,470-145,023$ & 12731 & 572,000 \\
\hline$\alpha$ Ara (79) I & $2008-217$ & 54.4 & $49.5-354.2$ & $94,195-157,552$ & 10626 & 24,600 \\
\hline$\alpha \operatorname{Ara~(79)~E~}$ & $2008-217$ & 54.4 & $49.5-70.2$ & $94,195-100,266$ & 2874 & 24,600 \\
\hline$\beta$ Cen (81) I \# & $2008-231$ & 66.7 & 294.4-267.6 & $72,829-151,692$ & 12191 & 546,000 \\
\hline$\beta$ Cen (85) I \# & $2008-260$ & 66.7 & $295.3-269.5$ & $73,112-143,414$ & 10991 & 531,000 \\
\hline$\alpha \operatorname{Ara}(85) \mathrm{I}$ & $2008-261$ & 54.4 & $49.7-353.3$ & $93,510-160,543$ & 10917 & 25,100 \\
\hline$\alpha \operatorname{Ara}(85) \mathrm{E}$ & $2008-261$ & 54.4 & 49.7-106.4 & $93,510-157,485$ & 10873 & 24,000 \\
\hline$\alpha \operatorname{Ara}(86) \mathrm{I}$ & $2008-268$ & 54.4 & $49.7-352.5$ & $93,407-163,471$ & 11220 & 22,400 \\
\hline$\alpha \operatorname{Ara~(86)~E~}$ & $2008-268$ & 54.4 & $49.7-107.2$ & $93,407-160,105$ & 11171 & 21,400 \\
\hline$\beta$ Cen (89) I \# & $2008-290$ & 66.7 & $296.4-269.8$ & $71,854-141,886$ & 10931 & 500,000 \\
\hline$\alpha$ Ara (90) I & $2008-298$ & 54.4 & $49.7-352.7$ & $92,106-160,553$ & 10949 & 20,200 \\
\hline$\alpha \operatorname{Ara}(90) \mathrm{E}$ & 2008-298 & 54.4 & $49.7-106.7$ & $92,106-156,650$ & 10812 & 20,000 \\
\hline$\alpha$ Cru (92) I \# & $2008-312$ & 68.2 & $125.0-181.6$ & $77,557-155,730$ & 20861 & 516,000 \\
\hline$\beta$ Cen (92) E \# & $2008-313$ & 66.7 & $42.7-59.1$ & $50,676-154,574$ & 16180 & 463,000 \\
\hline$\theta$ Нyа (94) I & $2008-332$ & 1.4 & $89.5-10.5$ & $83,453-436,956$ & 2994 & $1800^{*}$ \\
\hline$\theta$ Hya (94) E & $2008-332$ & 1.4 & $89.5-169.9$ & $83,453-500,646$ & 3446 & $1800^{*}$ \\
\hline$\beta$ Cen (96) I \# & $2008-343$ & 66.7 & $288.6-264.8$ & $72,456-155,341$ & 12582 & 441,000 \\
\hline$\alpha \operatorname{Ara}(96) \mathrm{I}$ & $2008-344$ & 54.4 & $46.9-1.6$ & $108,169-150,852$ & 9900 & 19,800 \\
\hline$\alpha \operatorname{Ara}(96) \mathrm{E}$ & $2008-344$ & 54.4 & $46.9-94.2$ & $108,169-155,151$ & 10551 & 19,300 \\
\hline
\end{tabular}


Table 1

(Continued)

\begin{tabular}{|c|c|c|c|c|c|c|}
\hline Occultation Star (rev) Side & Date (Year-Day) & $|B|(\mathrm{deg})$ & $\phi \operatorname{deg})$ & $R(\mathrm{~km})$ & Duration (s) & $I_{0}(\mathrm{~Hz})$ \\
\hline$\delta$ Cen (98) I \# & $2008-359$ & 55.6 & $209.3-212.0$ & $55,447-153,103$ & 14531 & 36,100 \\
\hline$\beta$ Cru (98) I & $2008-359$ & 65.2 & $157.3-202.6$ & $58,104-154,683$ & 18080 & 279,000 \\
\hline$\alpha \operatorname{Ara}(98) \mathrm{I}$ & $2008-360$ & 54.4 & $42.3-357.4$ & $110,536-154,532$ & 12407 & 17,200 \\
\hline$\alpha$ Ara (98) E & $2008-360$ & 54.4 & $42.3-86.3$ & $110,536-152,130$ & 12024 & 17,200 \\
\hline$\alpha$ Cru (100) I \# & 2009-012 & 68.2 & $124.2-164.8$ & $114,050-149,157$ & 14422 & 438,000 \\
\hline$\alpha$ Cru (100) E \# & 2009-012 & 68.2 & $124.2-83.5$ & $114,050-149,449$ & 14508 & 418,000 \\
\hline$\gamma$ Cas $(100) \mathrm{E}$ & 2009-015 & 66.3 & $86.4-66.0$ & $72,440-140,370$ & 9611 & 56,000 \\
\hline$\beta$ Cen (102) I \# & 2009-031 & 66.7 & $250.7-248.3$ & $73,243-143,508$ & 10311 & 369,000 \\
\hline$\beta$ Cen (104) I \# & 2009-053 & 66.7 & $179.2-220.0$ & $70,203-147,291$ & 27000 & 365,000 \\
\hline$\beta$ Cen (104) E \# & 2009-053 & 66.7 & $134.8-94.7$ & $68,934-131,988$ & 15591 & 365,000 \\
\hline$\varepsilon$ Cas (104) I & 2009-058 & 69.9 & $154.5-198.7$ & $111,694-154,568$ & 15864 & 4,400 \\
\hline$\varepsilon$ Cas (104) E & 2009-058 & 69.9 & $154.5-111.7$ & $111,694-151,532$ & 15197 & 4,400 \\
\hline$\theta$ Hya (104) I & 2009-062 & 1.4 & $89.3-3.0$ & $66,876-1051,956$ & 5513 & $1000^{*}$ \\
\hline$\theta$ Hya (104) E & 2009-062 & 1.4 & $89.3-172.5$ & $66,876-561,578$ & 2928 & $1000^{*}$ \\
\hline$\beta$ Cen (105) I \# & 2009-065 & 66.7 & $199.1-222.0$ & $88,502-158,713$ & 14561 & 310,000 \\
\hline$\beta$ Cen (105) E \# & 2009-065 & 66.7 & $121.7-91.5$ & $77,787-147,358$ & 15341 & 301,000 \\
\hline$\alpha \operatorname{Ara}(105) \mathrm{I}$ & 2009-066 & 54.4 & $39.2-343.9$ & $93,917-163,844$ & 19802 & 15,500 \\
\hline$\alpha \operatorname{Ara}(105) \mathrm{E}$ & 2009-066 & 54.4 & $39.2-88.6$ & $93,917-143,382$ & 15969 & 17,000 \\
\hline$\zeta$ Cen (112) I & $2009-163$ & 53.6 & $236.6-241.2$ & $71,486-143,214$ & 16464 & 53,000 \\
\hline$\mu$ Cen (113) I & $2009-177$ & 48.7 & $236.2-240.6$ & $75,975-155,785$ & 16090 & 9,400 \\
\hline$\alpha \operatorname{Lup}(113) \mathrm{I}$ & 2009-178 & 53.8 & $172.4-217.8$ & $83,839-118,956$ & 20269 & 26,400 \\
\hline$\alpha \operatorname{Lup}(113) \mathrm{E}$ & $2009-178$ & 53.8 & $172.4-160.9$ & $83,839-85,541$ & 4071 & 26,400 \\
\hline$\beta \operatorname{Lup}(114) \mathrm{I}$ & 2009-193 & 49.6 & $186.8-217.4$ & $118,474-144,836$ & 16090 & $24,000^{*}$ \\
\hline$\lambda \operatorname{Sco}(114) \mathrm{I} \#$ & 2009-195 & 41.7 & $219.6-259.1$ & $110,857-148,227$ & 31840 & 88,500 \\
\hline$\sigma \operatorname{Sgr}(114) \mathrm{I} \#$ & $2009-198$ & 29.1 & $332.1-329.2$ & $84,449-149,875$ & 21040 & 33,300 \\
\hline$\mu \operatorname{Sgr}(115) \mathrm{I}$ & $2009-212$ & 24.9 & $44.1-27.4$ & $90,967-94,935$ & 10104 & $>200+$ \\
\hline$\mu \operatorname{Sgr}(115) \mathrm{E}$ & 2009-212 & 24.9 & $44.1-80.3$ & $90,967-112,618$ & 24707 & $>200+$ \\
\hline$\beta$ Per (116) I & 2009-223 & 47.4 & $153.8-169.6$ & $131,436-135,217$ & 2294 & $2000^{*}$ \\
\hline$\beta$ Per (116) E & 2009-223 & 47.4 & $153.8-138.5$ & $131,436-134,741$ & 2206 & $2200^{*}$ \\
\hline$\alpha \operatorname{Vir}(116) \mathrm{I}$ & $2009-223$ & 17.2 & $245.2-241.9$ & $103,059-144,566$ & 3764 & 165,000 \\
\hline$\pi 4$ Ori (117) E & 2009-239 & 3.7 & $105.2-109.1$ & $68,168-145,300$ & 4811 & 3300 \\
\hline$\alpha \operatorname{Vir}(124) \mathrm{E}$ & $2010-011$ & 17.2 & $121.7-124.1$ & $70,494-142,431$ & 6011 & 165,000 \\
\hline$\beta \operatorname{Lib}(124) \mathrm{E}$ & $2010-011$ & 15.8 & $236.9-221.5$ & $115,071-123,509$ & 2756 & 2900 \\
\hline
\end{tabular}

Notes. Rev refers to the number of the orbit of Cassini around Saturn on which the observation occurred. Cassini revs are numbered 0, A, B, C, 3, 4, and consecutively thereafter. Ranges in $\phi$ and $R$ are for the entire observation, and the range in $\phi$ is listed in the order corresponding to the range in $R$. In some cases, part of the occultation is obscured by the planet. The values listed here are for the full occultation, not just the part where the star is unobstructed. Values of $I_{0}$ are approximate mean values. See the text for a discussion of variations in $I_{0}$. Durations indicate the time from the start of measurements to the last measurement, including gaps caused by data dropouts. "** indicates occultations for which the background cannot be directly measured, and the values listed in the $I_{0}$ column are $I_{0}+b$ for those occultations. "+" indicates occultations for which the stellar signal cannot be directly measured, and the values listed in the $I_{0}$ column are estimates from other occultations. "\#" indicates that there is a detectable signal from more than one star in the data. I and $\mathrm{E}$ indicate ingress and egress occultations, respectively.

profiles. This can be seen, for example, in the difference in the photon count rate between the ingress and egress portions of the $\alpha$ Virginis occultation in the A ring (Figure 1). The angle, $\phi$, between the radial direction at the point where the line of sight pierces the ring plane and the projection of the occultation line of sight onto the ring plane determines the extent to which light can pass between self-gravity wakes at a given ring radius and elevation angle $B$ (Colwell et al. 2006, 2007; Hedman et al. 2007a; Nicholson \& Hedman 2009).

In addition to the variation in sensitivity of the HSP during a single occultation, the average sensitivity of the detector has been declining with use over the course of the mission. The sensitivity has declined linearly with total accumulated counts, with most of the absolute decline occurring during the high-inclination sequence that concluded the Cassini Prime mission and began the Cassini Equinox mission, from early 2008 through early 2009. Thus, absolute counting rates for individual stars and minimum background values have declined over the course of the mission so that each occultation must be individually calibrated. This can be seen in Table 1, for example, by comparing the values of $I_{0}$ for $\beta$ Cen, one of the brightest UV stars we observe, from rev 77 through rev 105 where there is roughly a $50 \%$ reduction in sensitivity ("rev" identifies the number of the orbit or revolution of Cassini around Saturn). Table 1 lists the ring stellar occultations observed by UVIS through rev 114. Data were also recorded with the HSP during some VIMS stellar occultations, but in most cases no stellar signal was detected by the HSP from those cool stars so those observations are not included in Table 1.

\section{CALIBRATION AND PDS DATA PRODUCT}

In order to generate a full profile of $\tau_{n}$ from the data, profiles of $b$ and $I_{0}$ are constructed by linearly interpolating between regions where each value can be measured directly, or by extrapolation. The data are recorded as a time series with a constant integration period. One approach for interpolation and extrapolation of $b$ and $I_{0}$ would be to work in the original uniformly sampled time domain of the data. The approach we 
Table 2

Opaque Regions for Background Determination

\begin{tabular}{ccc}
\hline \hline Number & Inner Radius $(\mathrm{km})$ & Outer Radius $(\mathrm{km})$ \\
\hline 1 & 100,190 & 100,210 \\
2 & 100,260 & 100,280 \\
3 & 100,750 & 100,770 \\
4 & 101,780 & 101,800 \\
5 & 102,090 & 102,110 \\
6 & 103,870 & 103,890 \\
7 & 104,460 & 104,480 \\
8 & 104,860 & 104,880 \\
9 & 105,390 & 105,410 \\
10 & 106,100 & 106,150 \\
11 & 107,870 & 107,900 \\
\hline
\end{tabular}

Table 3

Gap Regions for Determination of $I_{0}$

\begin{tabular}{lcc}
\hline \hline Name & Inner Radius $(\mathrm{km})$ & Outer Radius $(\mathrm{km})$ \\
\hline D Ring & 74,420 & 74,450 \\
G1 & 75,760 & 75,800 \\
Colombo & 77,770 & 77,820 \\
Maxwell & 87,360 & 87,400 \\
Dawes & 90,213 & 90,215 \\
Huygens & 117,720 & 117,760 \\
Herschel & 118,200 & 118,210 \\
Herschel & 118,270 & 118,280 \\
Russell & 118,605 & 118,625 \\
Jeffreys & 118,935 & 118,955 \\
Laplace & 119,860 & 119,950 \\
Barnard & 120,310 & 120,312 \\
Encke & 133,500 & 133,700 \\
Keeler & 136,495 & 136,505 \\
Roche & 137,000 & 137,500 \\
\hline
\end{tabular}

Note. Subsets of the gaps were used to avoid eccentric edges and ringlets within the gaps. Full gap boundaries are in Colwell et al. (2009).

adopt is to calculate the location in the ring plane of each data point and then interpolate and extrapolate in the ring plane radius domain. The reason for this choice is that it is variations in the radial structure of the rings that is primarily responsible for variations in $b$ and $I_{0}$, rather than temporal variations. The rampup instrumental response described in Section 2 and in Colwell et al. (2007) depends both on time and on the history of the signal into the instrument. For ring occultations, this signal is most strongly dependent on structure in the rings. The first step in our data processing is therefore the calculation of the ring intercept point of each sample in the occultation data.

\subsection{Calculation of Occultation Geometry}

The calculation of occultation geometry is described in detail in French et al. (1993), and we follow that general procedure closely. Here, we summarize the steps we follow to calculate the occultation intercept points. We get the spacecraft trajectory from SPICE kernels at the Navigation and Ancillary Information Facility (NAIF) of the PDS. We use ephemeris kernels with combined spacecraft and planetary state data based on reconstruction of the spacecraft trajectory by the Cassini navigation team. Stellar coordinates, proper motions, and parallax are taken from the Hipparcos catalog and are propagated forward from the Hipparcos epoch of 1991.25 to the time of the occultation and corrected for parallax to the location of Cassini and for aberration due to motion of Saturn with

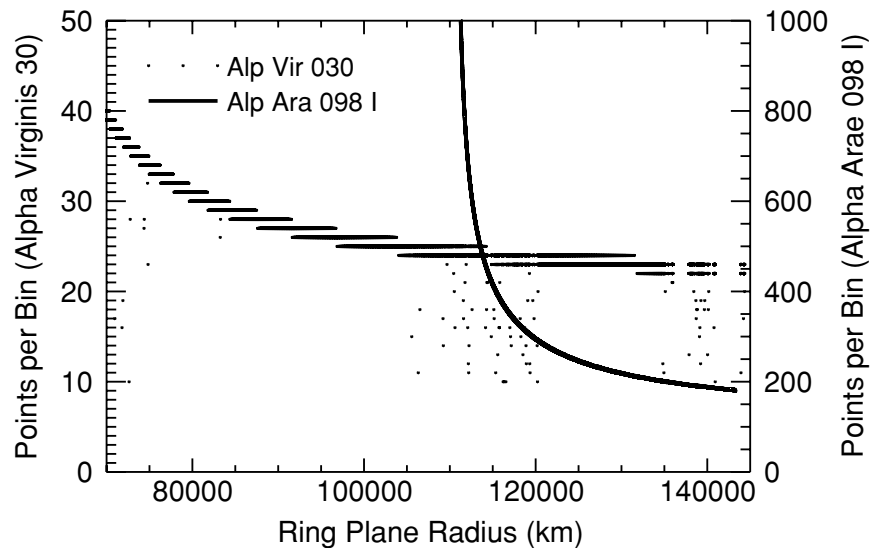

Figure 2. Number of HSP integration periods making up a $1 \mathrm{~km}$ radial bin for a radial occultation ( $\alpha$ Virginis Rev 30) and a chord occultation ( $\alpha$ Arae Rev 98). The minimum radius of the $\alpha$ Arae Rev 98 occultation was $110,536 \mathrm{~km}$, and at that location the number of integrations in a $1 \mathrm{~km}$ bin is over 50,000. The scatter in the number of points per bin for the $\alpha$ Vir occultation is due to a large number (104) of data dropouts in that occultation.

respect to the solar system barycenter. The ring plane is assumed to be exactly normal to the pole of Saturn which is retrieved from NAIF planetary constants kernels for the time of the occultation. We make a non-iterative correction for general relativity treating Saturn as a sphere. The general relativistic correction is on the order of $10 \mathrm{~m}$ or less. The primary uncertainties are in the position of the spacecraft along its trajectory and the pole direction of Saturn. Based on comparisons of different occultation profiles to features that are circular or nearly so (Nicholson et al. 1990; French et al. 1993), these errors are on the order of $1 \mathrm{~km}$. Ring longitudes are referenced to the ascending node of the ring plane on the $\mathrm{J} 2000$ inertial $X-Y$ plane.

\subsection{Binning the Data}

We next bin the data to a uniform radial scale. The raw data typically have radial sampling intervals of several meters to a few tens of meters, though for occultations that cut a chord across the rings, the radial distance between points essentially vanishes at the minimum ring plane radius sampled by the occultation. To facilitate the intercomparison of occultations and the generation of model profiles of $b$ and $I_{0}$, we rebin the data to a radial resolution of $1 \mathrm{~km}$. This process can introduce a small error in the number of counts because we are specifying the new bin boundaries to be separated by exactly $1 \mathrm{~km}$, and the boundaries of the raw data measurements in general do not coincide with the new boundaries. When there are 10 or more raw integration periods within the $1 \mathrm{~km}$ bins, we sum the points whose boundaries lie closest to the $1 \mathrm{~km}$ bin boundaries. If there are fewer points, we perform a linear interpolation between the two points that straddle the bin boundary and divide the counts in the raw data bin that straddles the boundary of the $1 \mathrm{~km}$ bin proportionately between the two adjacent $1 \mathrm{~km}$ bins. Figure 2 shows the number of integration periods in $1 \mathrm{~km}$ bins for two occultations. The lower-resolution $\alpha$ Virginis rev 030 occultation shows bin-to-bin scatter in the number of integration periods within a bin as well as the effects of numerous data dropouts for this observation (due to poor weather at the Deep Space Network receiving station). The $\alpha$ Arae rev 098 occultation was a grazing, or chord, occultation, so the number of points within a $1 \mathrm{~km}$ radial bin is very large at the minimum sampled radius. 


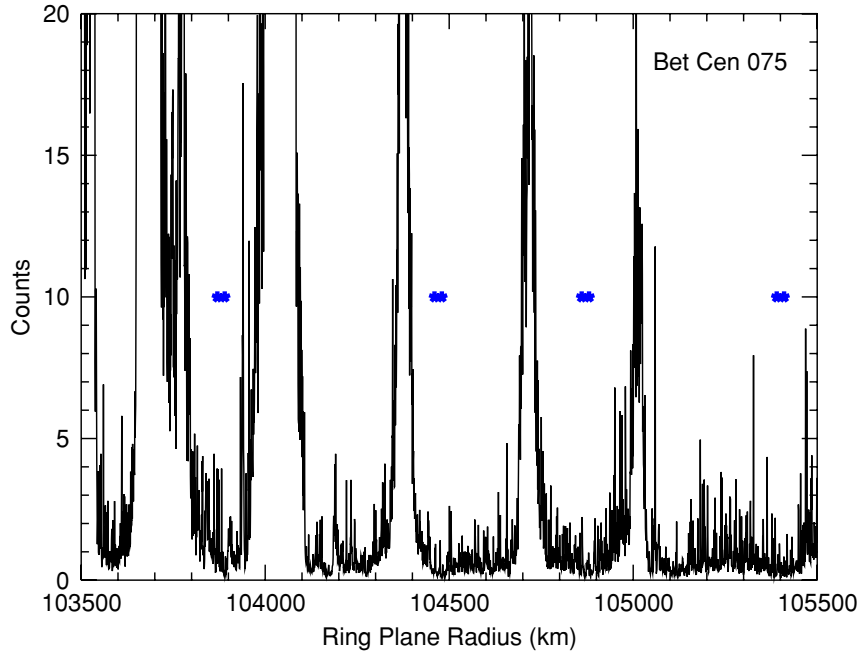

Figure 3. Data from the $\beta$ Cen Rev 75 occultation showing the central B ring and indicating (asterisks) some of the regions used for determination of the background level (Table 2).

(A color version of this figure is available in the online journal.)

\subsection{Determination of $b$ and $I_{0}$}

We next identify regions where the star is completely occulted to determine the background photon count rate, $b$. Parts of the $\mathrm{B} 2$ and $\mathrm{B} 3$ regions of the $\mathrm{B}$ ring have shown no transmitted signal in any occultation (Colwell et al. 2009). The regions we use to determine the background are listed in Table 2. Even in these opaque regions there are hints of structure, but the photon count rates there are reasonable estimates of the background (Figure 3). Determination of $b$ in the $\mathrm{C}$ and $\mathrm{A}$ ring regions requires either additional data or perfect knowledge of $I_{0}$ and $\tau$, so we assume a constant value extrapolated from the B ring region. The Titan ringlet in the inner $\mathrm{C}$ ring appears to have narrow opaque regions in the core, interrupted by small-scale $(\sim 1 \mathrm{~km})$ structure, but we do not include this in our standard estimation of $b$. It is also possible to determine $b$ in regions outside the $\mathrm{B}$ ring with simultaneous measurements of the ring UV reflectance with the FUV channel. Colwell et al. (2007) did this for some occultations in their analysis of self-gravity wakes. Their procedure was to measure the FUV signal as a function of ring plane radius and use that as a guide for the radial dependence of the background photon count rate. The background was then normalized by the measured values in opaque regions of the ring. The measured FUV signal on the rings is a combination of scattered sunlight and Ly $\alpha$ transmitted through the rings. FUV data are not available for all occultations, however, and there are complications associated with interpreting FUV data taken during stellar occultations when the star is in the FUV field of view. Some of the star signal gets mislocated onto other rows (see Esposito et al. 1998, 2004 for detailed descriptions of the instrument and its different operational modes). If that signal is attributed to ring or $\operatorname{Ly} \alpha$ background, then the background signal would be overestimated.

For those occultations that have coincident FUV data, we derive a radial profile of the FUV signal at $4000 \mathrm{~km}$ radial resolution by removing the data that show the signal from the star. This radial profile is obtained from data taken at different longitudes than the HSP occultation data, so the azimuthal asymmetries in ring brightness due to self-gravity wakes (e.g., Franklin and Colombo 1978; Porco et al. 2008) create additional complications in using the FUV data for the background

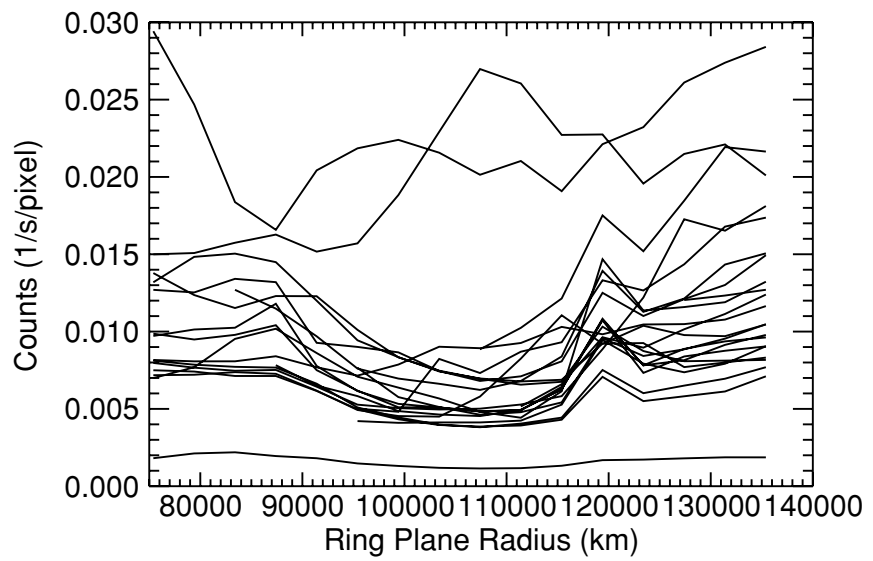

Figure 4. Measured FUV signal for several occultations with the signal from detector rows 28-36 removed. The observations shown are primarily for the unlit face of the rings where $\operatorname{Ly} \alpha$ is the dominant source of background, leading to minimum signal in the opaque B ring and larger signals in the more transparent ring regions. Contamination of the signal from the star is also an issue in those regions due to instrument's spatial point spread function.

determination. Because of the complications involved in using FUV data for background determination, mentioned above, and because of the partially subjective nature of adapting the FUV profile to the HSP background model, we do not use the FUV data for our standard model of $b$. Instead we provide the derived FUV radial profile to the PDS for users who wish to use it to develop their own model of $b$. Examples of the FUV radial profiles are shown in Figure 4.

The image of the star falls near the center of the field of view for the FUV channel (spatial rows 31 and 32, where the rows are numbered 0 to 63 along the UVIS slit). The instrument spatial point spread function causes some of the starlight to bleed over into nearby pixels, so we edit out data in rows 28-36 to remove most of the starlight. The remaining sources of counts in the FUV are Ly $\alpha$, scattered sunlight, and scattered Saturn-shine. Counts from each of these vary depending on the observational geometry and solar flux levels. For each spatial pixel, we average the raw counts over the spectral pixels in three wavelength ranges from 1118-1911 $\AA$ (the full extent of the spectrum), 1700-1911 $\AA$ (the long-wavelength portion of the spectrum), and 1199-1224 $\AA$ (Ly $\alpha)$. We use raw counts because the sensitivity of the FUV detector with wavelength is close to that of the HSP. Thus, the raw signals recorded by both the FUV and the HSP are roughly proportional to each other. We divide the rings into $4000 \mathrm{~km}$ radial bins from the inner $\mathrm{C}$ ring to the outer A ring. We then determine the projected field of view of each spatial pixel on the ring plane for each of the four corners of the pixel at the initial and final times for an integration period and determine a minimum and maximum ring plane radius for that pixel over the course of the integration period. For each radial bin, we average the spectral averages for all pixels where their minimum and maximum radial extent falls within the bin, resulting in three count levels for each radial bin, one for each of the three wavelength ranges.

The minimum background in the HSP has been established by a number of observations of the shadowed rings and dark sky (Chambers et al. 2008) as well as observations of VIMS stellar occultations. The HSP count rates in these observations were typically between 100 and 150 counts $\mathrm{s}^{-1}$ in measurements made in 2004-2005. The signal observed from stars ranges from a few thousand counts per second to several hundred thousand counts per second. In some cases, the scattered sunlight 
background signal can be up to $\sim 10$ times higher than the dark sky count rate and can be a significant fraction of the overall signal for faint stars. Although it is not a practical concern because we analyze each occultation independently, it is important to remember that the overall HSP sensitivity is declining with total accumulated exposure such that the background levels observed later in the mission are at lower absolute counting rates than those reported in Chambers et al. (2008) and Colwell et al. (2007).

Our standard procedure for estimating $b$ outlined here, based only on opaque regions in the central B ring, likely underestimates $b$ elsewhere in the rings because we simply assume a constant value of $b$ from the B ring regions to the edges of the ring system. FUV data, where available, and the signal in apparently opaque regions of the Titan ringlet suggest that the background may roughly double from the central $\mathrm{B}$ ring to the inner $\mathrm{C}$ ring and to the outer A ring (Figure 4). For many occultations the background signal is negligible, and the derived optical depths are insensitive to systematic uncertainties in the background. Drifts in the instrument's sensitivity within an occultation make it difficult to identify broad, low-optical-depth features within gaps and also complicate the determination of the appropriate value of the star brightness to use.

The unobstructed stellar photon count rate, $I_{0}$, is modeled by taking measurements in transparent regions in the rings where the observed photon count rate is $I_{0}+b$ and subtracting the modeled background photon count rate. We use linear interpolation between the gaps in the rings and assume a constant value of $I_{0}$ beyond the innermost and outermost gaps. Within some of the broader gaps, notably the Huygens Gap and the Encke Gap, the observed photon count rate changes noticeably due to the changing detector response (e.g., Figure 1). We do not model this changing signal within a gap; we use the simpler technique of taking an average gap value and doing linear interpolation between gaps so that the instrumental effect is clear and we do not risk convolving the effects of broad faint rings, known to exist in some gaps, with our modeling of $I_{0}$. We use gaps in the A ring, $\mathrm{C}$ ring, and Cassini Division, as well as the Roche Division between the A and F rings, and the optically thin outer D ring (exterior to the D73 ringlet) to determine $I_{0}$ (Table 3 ). When the nominal geometric solution of an occultation leads to a misalignment of the gap with our nominal ranges, we manually adjust the fit to use only data actually within the gap.

\subsection{Estimated Maximum Detectable Optical Depth}

With the data binned to a common $1 \mathrm{~km}$ radial grid and with a model of both $I_{0}$ and $b$, we use Equation (3) to generate a normal optical depth profile. We select $\tau_{\max }$ to be the largest normal optical depth that has a finite $1 \sigma$ upper error bar. The upper error bar in the measured normal optical depth, $\tau_{\mathrm{n}}$, based only on counting statistics (and not on systematic uncertainties in modeling $I_{0}$ and $b$ as described above) is given by (Colwell et al. 1990)

$$
\tau_{+}=\mu \ln I_{0}-\mu \ln \left[I_{0} e^{-\tau_{n} / \mu}-Z \sqrt{I_{0} e^{-\tau_{n} / \mu}+b}\right],
$$

where the quantity under the radical is simply the measured photon count rate $I, Z$ is the number of standard deviations for the error bar, and we use $Z=1$. Our criterion for $\tau_{\max }$ is thus obtained from requiring the term in square brackets in Equation (4) to be positive, or

$$
\tau_{\max }=\mu \ln \left[I_{0} / \sqrt{I}\right]
$$

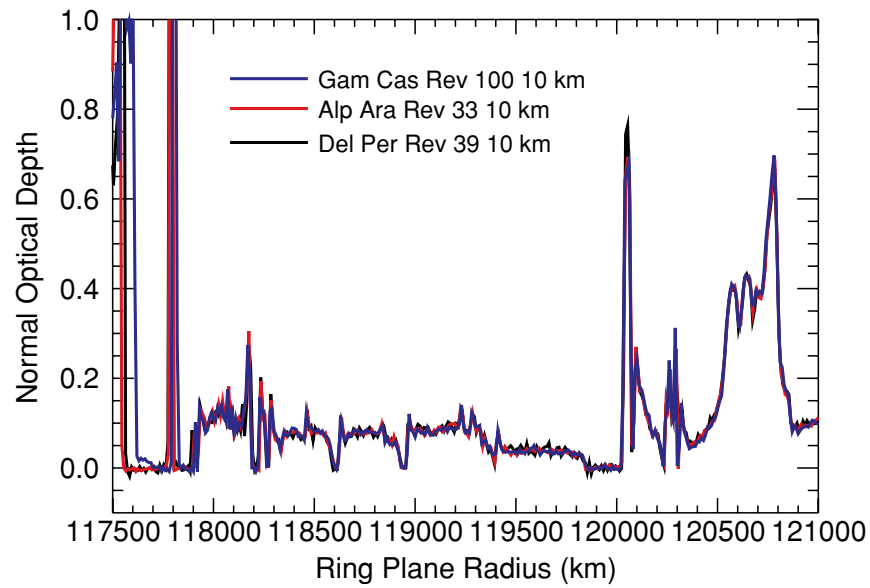

Figure 5. Three optical depth profiles of the Cassini Division at $10 \mathrm{~km}$ resolution. The optical depth in the Huygens Gap is not uniformly zero in the $\gamma$ Cas Rev 100 occultation profile due to the instrumental effects described in the text. Each of these three stars is far above the ring plane $\left(B>54^{\circ}\right.$, Table 1$)$, so the effects of self-gravity wakes on the measured optical depth are not noticeable.

Because the measured photon count rate, I, can be zero, we also require the denominator in Equation (5) to be at least 1 , representing one photon count in the bin. As the spatial resolution is degraded by increasing the number of points, $n$, in a bin, the value of $\tau_{\max }$ increases roughly as $\ln \sqrt{n}$. The fractional increase in $\tau_{\max }$ as the resolution is decreased depends on the numerical values of $I, I_{0}$, and $n$. Furthermore, in order to assign a complete confidence level to any particular measurement of $\tau_{\mathrm{n}}$, systematic uncertainties in both $b$ and $I_{0}$ must be estimated and combined with the statistical uncertainty introduced by low counting rates in high optical depth regions (see the discussion in Colwell et al. 2007).

Uncertainties in the calculated optical depths are smallest on an absolute basis at low optical depths, but are smallest in relative terms at intermediate values of the optical depth, with the precise value depending on the brightness of the star. The relative error in $\tau_{\mathrm{n}}$ has a broad minimum, is proportional to $\sqrt{I_{0}}$ for $\tau_{\mathrm{n}}$ not close to $\tau_{\max }$, and is less than a few percent for $\tau_{\mathrm{n}}$ between 0.1 and $\sim \tau_{\max } / 2$ if $I_{0}>10^{5}$. The minimum relative error is at a line-of-sight optical depth of $\tau=2$.

\subsection{Calibrated PDS Optical Depth Profiles}

The calibrated data are delivered to the PDS at $1 \mathrm{~km}$ and $10 \mathrm{~km}$ resolutions on identical radial grids so that occultations can be directly compared to each other. The delivered data products include $\tau_{\max }$ and $\tau_{\mathrm{n}}$ so that whenever $\tau_{\mathrm{n}}=\tau_{\max }$ in the data, the user knows that this is essentially a lower limit on $\tau_{\mathrm{n}}$. In addition to the optical depth, the data files include the ring plane radius, inertial ring longitude, and ephemeris time (relative to J2000) at the center of the bin. The number of raw data points included in the bin $(n)$ and the value of the raw data are also included, as is the model value for $I_{0}$ and $b$. This provides enough information for a user to use the calibration model to resample the data to arbitrary resolution, including full resolution, and to experiment with modified models for $I_{0}$ and $b$. The dataset ID in the PDS is CO-SR-UVIS-2/4-OCC-V1.0.

Examples of radial optical depth profiles calculated following the procedure described above at $1 \mathrm{~km}$ and $10 \mathrm{~km}$ radial resolution are shown in Figures 5-9. The central part of the $\mathrm{B}$ ring contains regions which remain opaque to our brightest, 


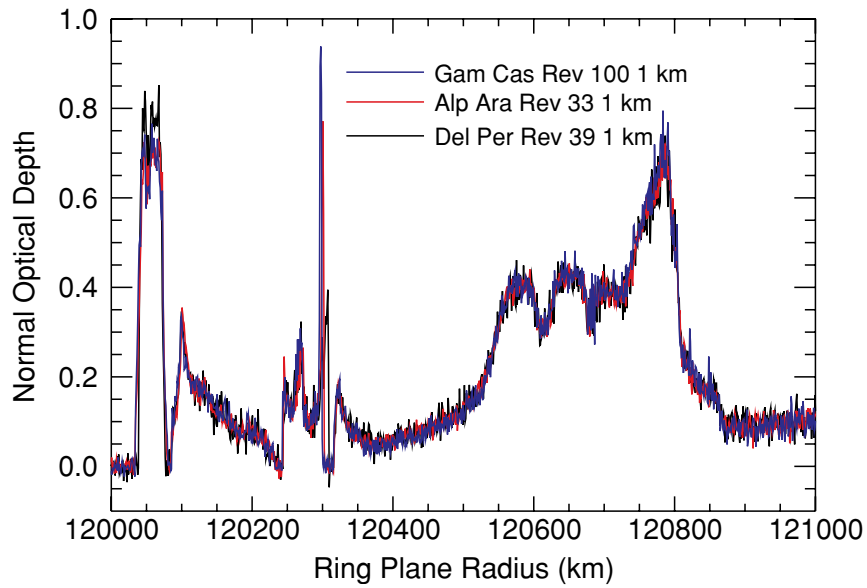

Figure 6. Three optical depth profiles of the outer Cassini Division at $1 \mathrm{~km}$ resolution from the same occultation profiles as shown in Figure 5.

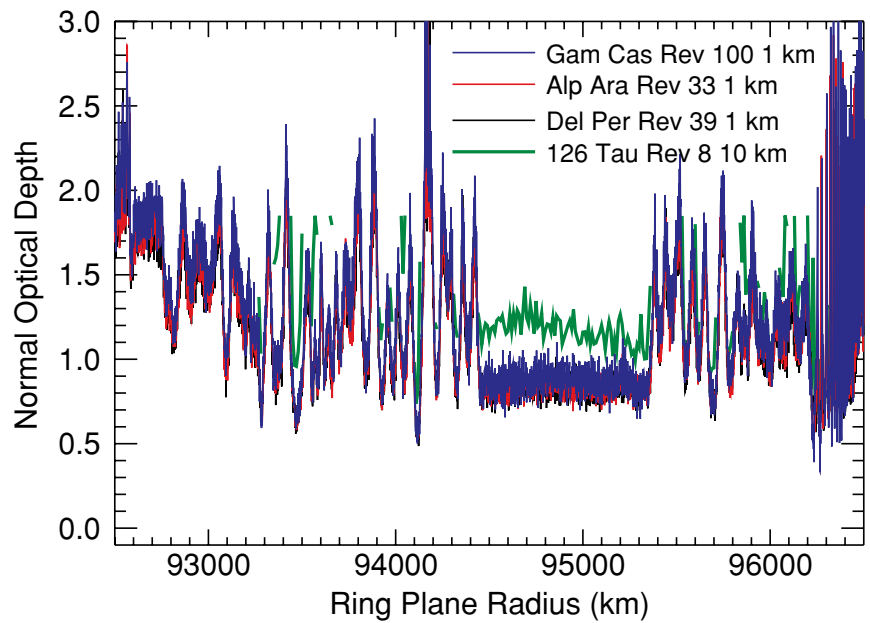

Figure 7. Comparison of optical depth profiles in the inner B ring at 1 and $10 \mathrm{~km}$ from four different occultations. The star 126 Tauri has $B=21^{\circ}$. 1 , so the presence of self-gravity wakes affects the measured optical depth resulting in a noticeable difference between the profile for that star and the other three that are closer to normal incidence (Colwell et al. 2007).

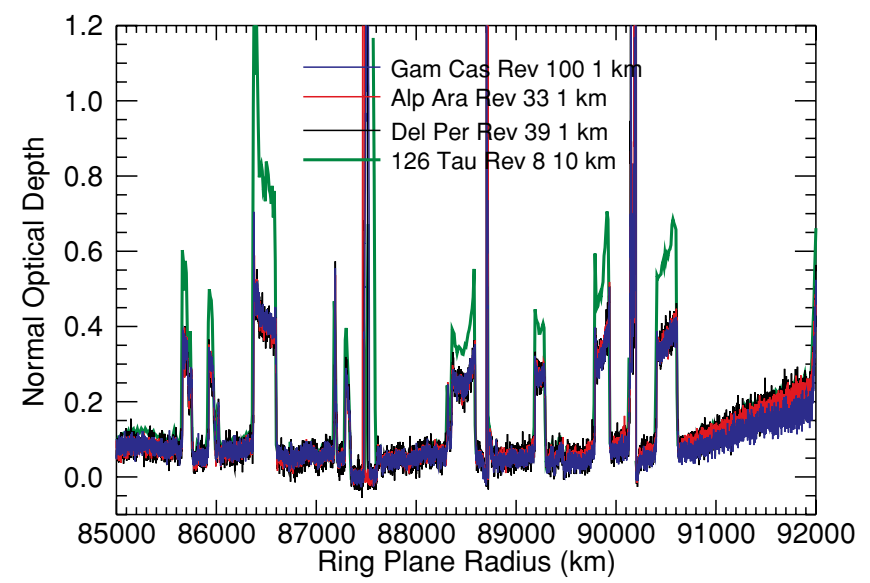

Figure 8. Four optical depth profiles of the outer $\mathrm{C}$ ring using the same occultations as shown in Figure 5. The 126 Tauri is shown at $10 \mathrm{~km}$ resolution because the star is fainter than the other three. There is a smaller offset in the low- $\tau$ regions that may indicate that the background for the 126 Tauri is overestimated (see also Colwell et al. 2007 for a detailed discussion of this occultation and its complicated background).

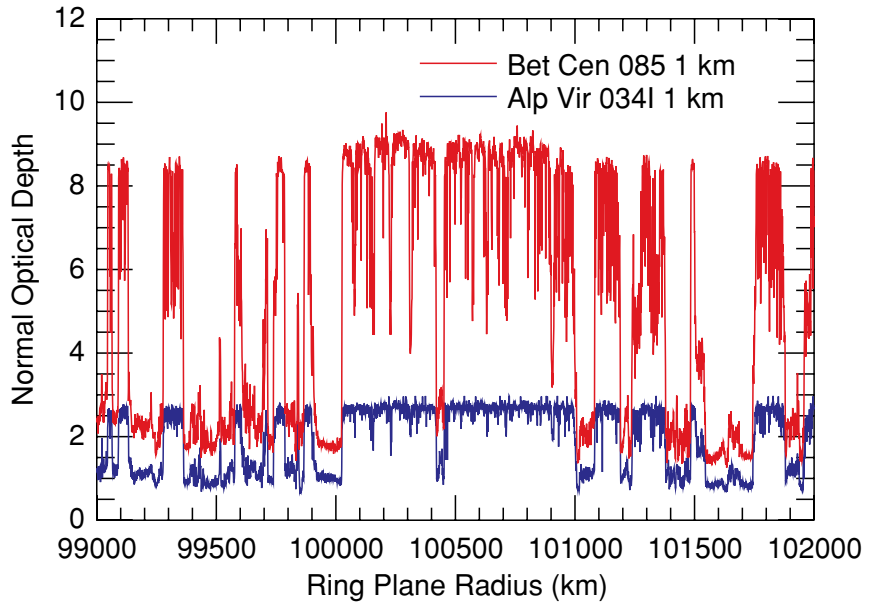

Figure 9. Optically thick region of the B ring seen in two occultations at very different incidence angles. The high incidence angle $\beta$ Centauri occultation is able to measure much larger normal optical depths, and this region of the rings remains opaque at this geometry: $\tau=\tau_{\max }$ for both stars over much of this and other regions in the B ring. Where $\tau$ is measured, it differs between the two stars due to the effect of self-gravity wakes (Colwell et al. 2007). Some high optical depth structure that is only suggested by the $\alpha$ Virginis occultation shows up at $\tau \sim 5$ in the $\beta$ Centauri occultation.

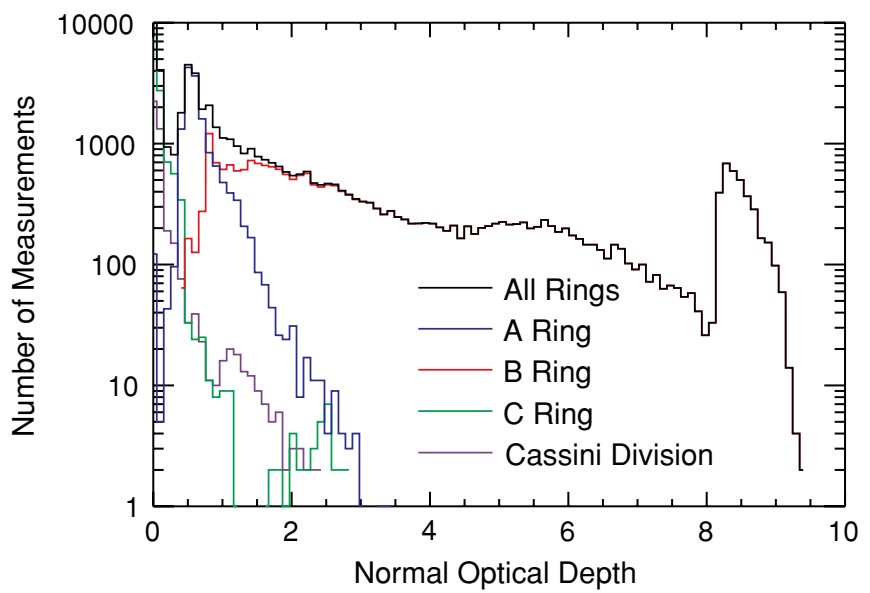

Figure 10. Histograms of optical depth measurements from the $\beta$ Centauri (Rev 085; see Figure 9) occultation. The peak at large optical depth reflects $\tau_{\max }$. A smaller, broader peak at $\tau=5-6$ reflects real measurements of transmitted starlight in the central B ring.

highest-incidence angle stars (Figure 9). Figure 10 shows the distribution of measured normal optical depths from an occultation of $\beta$ Centauri $(B=66.7)$. There is a shallow distribution of $\tau_{\mathrm{n}}$ in the $\mathrm{B}$ ring and a significant number of measurements where $\tau_{\mathrm{n}}=\tau_{\max }(5.3 \%$ of the total number of ring measurements and $13 \%$ of all measurements in the $\mathrm{B}$ ring at $1 \mathrm{~km}$ resolution). Only the $\mathrm{B}$ ring has these opaque regions in the brightest star occultations observed by UVIS.

\section{SUMMARY}

The Cassini UVIS has observed over 100 stellar occultations by Saturn's rings with its HSP through the first 6 years of its orbital tour of the Saturn system. These occultations span a variety of viewing geometries and star brightnesses (Table 1). Occultations at different viewing geometries can reveal vertical and non-axisymmetric structure in the rings. Even occultations of bright stars at high incidence angle reveal significant regions in the $\mathrm{B}$ ring that are opaque, with a nominal minimum optical depth $\sim 10$ at $1 \mathrm{~km}$ resolution. 
Comparison of the optical depth of the rings (or equivalently the transparency) between occultations in order to extract information about this structure requires careful consideration of instrumental effects and background signal, as well as a resampling of the data to a common radial sampling grid. We describe here a standard procedure for producing optical depth profiles from the UVIS HSP star occultations. These profiles are delivered to the PDS as they are generated. Researchers can resample the data to any desired resolution using the data products provided which include models of the background and star intensity, as well as supplementary data on the ring reflectivity for use in deriving more refined background models.

This work was supported by NASA through the Cassini project and by the Cassini Data Analysis Program, grants NNX08AQ73G and NNX10AF20G. We appreciate the detailed review of an anonymous referee whose comments improved the paper. We thank the entire Cassini Rings Target Working Team and in particular Jeff Cuzzi, Phil Nicholson, Kelly Perry, and Brad Wallis whose efforts made these observations possible.

\section{REFERENCES}

Albers, N., Sremčević, M., Colwell, J. E., \& Esposito, L. W. 2010, Icarus, submitted

Beurle, K., Murray, C. D., Williams, G. A., Evans, M. W., Cooper, N. J., \& Agnor, C. B. 2010, ApJ, 718, L176

Chambers, L. S., Cuzzi, J. N., Asphaug, E., Colwell, J. E., \& Sugita, S. 2008, Icarus, 194, 623

Charnoz, S., Porco, C. C., Déau, E., Brahic, A., Spitale, J. N., Bacques, G., \& Baillié, K. 2005, Science, 310, 1300
Colwell, J. E., Esposito, L. W., \& Sremčević, M. 2006, Geophys. Res. Lett., 33, L07201

Colwell, J. E., Esposito, L. W., Sremčević, M., Stewart, G. R., \& McClintock, W. E. 2007, Icarus, 190, 127

Colwell, J. E., Jerousek, R. G., \& Esposito, L. W. 2008, BAAS, 40, 443

Colwell, J. E., Nicholson, P. D., Tiscareno, M. S., Murray, C. D., French, R. G., \& Marouf, E. A. 2009, in Saturn from Cassini-Huygens, ed. M. K. Dougherty, L. W. Esposito, \& S. M. Krimigis (Dordrecht: Springer), 375

Colwell, J. E., et al. 1990, Icarus, 83, 102

Esposito, L. W., Colwell, J. E., \& McClintock, W. E. 1998, Planet. Space Sci., 46, 1221

Esposito, L. W., Meinke, B. K., Colwell, J. E., Nicholson, P. D., \& Hedman, M. M. 2008, Icarus, 194, 278

Esposito, L. W., et al. 2004, Space Sci. Rev., 115, 299

Franklin, F. A., \& Colombo, G. 1978, Icarus, 33, 279

French, R. G., McGhee, C. A., Dones, L., \& Lissauer, J. J. 2003, Icarus, 162, 143

French, R. G., et al. 1993, Icarus, 103, 163

Hedman, M. M., Nicholson, P. D., Salo, H., Wallis, B. D., Buratti, B. J., Baines, K. H., Brown, R. H., \& Clark, R. N. 2007a, AJ, 133, 2624

Hedman, M. M., et al. 2007b, Icarus, 188, 89

Murray, C. D., Beurle, K., Cooper, N. J., Evans, M. W., Williams, G., \& Charnoz, S. 2008, Nature, 453, 739

Murray, C. D., Chavez, C., Beurle, K., Cooper, N., Evans, M. W., Burns, J. A., \& Porco, C. C. 2005, Nature, 437, 1326

Nicholson, P. D., Cooke, M. L., \& Pelton, E. 1990, AJ, 100, 1339

Porco, C. C., Weiss, J. W., Richardson, D. C., Dones, L., Quinn, T., \& Throop, H. 2008, AJ, 136, 2172

Sremčević, M., Schmidt, J., Salo, H., Seiss, M., Spahn, F., \& Albers, N. 2007, Nature, 449, 1019

Thomson, F. S., Marouf, E. A., Tyler, G. L., French, R. G., \& Rappaport, N. J. 2007, Geophys. Res. Lett., 34, L24203

Tiscareno, M. S., Burns, J. A., Hedman, M. M., \& Porco, C. C. 2008, AJ, 135, 1083

Tiscareno, M. S., Burns, J. A., Hedman, M. M., Porco, C. C., Weiss, J. W., Dones, L., Richardson, D. C., \& Murray, C. D. 2006, Nature, 440, 648

Tiscareno, M. S., Burns, J. A., Nicholson, P. D., Hedman, M. M., \& Porco, C. C. 2007 , Icarus, 189,14 